\title{
Imatinib-induced hyperbilirubinemia with UGT1A1 (*28) promoter polymorphism: first case series in patients with gastrointestinal stromal tumor
}

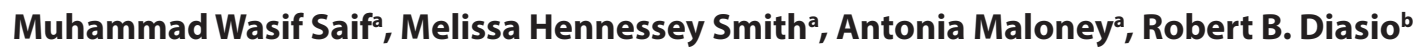 \\ Tufts Cancer Center-Tufts Medical Center, Boston, MA; Mayo Clinic Cancer Center, Rochester, MN, USA
}

\section{Abstract}

\section{Introduction}

Imatinib has revolutionized the treatment of chronic myeloid leukemia (CML) and gastrointestinal stromal tumor (GIST). It is an orally administered protein-tyrosine kinase inhibitor (TKI), an enzyme produced by BCR-ABL fusion which results from translocation of 9:22 chromosome (Philadelphia chromosome) [1]. Imatinib blocks proliferation and induces apoptosis of BCR-ABL-expression in CML [2].

${ }^{a}$ Division of Hematology/Oncology and Experimental Therapeutics, Tufts Cancer Center- Tufts Medical Center, Boston, MA, USA (Muhammad Wasif Saif, Melissa Hennessey Smith, Antonia Maloney), ${ }^{\mathrm{b}}$ Mayo Clinic Cancer Center, Rochester, MN (Robert B. Diasio), USA

\section{Conflict of Interest: None}

Correspondence to: Muhammad Wasif Saif, MD, MBBS, Division of Hematology/Oncology and Experimental Therapeutics, Tufts Medical Center, 800 Washington Street Box: 245, Boston, MA 02111, USA, Tel.: + 1617636 5627, Fax: +1 617636 8538,

e-mail:wsaif@tuftsmedicalcenter.org

Received 23 March 2016; accepted 12 May 2016: published online 10 June 2016

DOI: http://dx.doi.org/10.20524/aog.2016.0053
It also has comparable activity against the KIT receptor (wild type and mutant) and platelet-derived growth factor receptor [3]. Imatinib works as a competitive antagonist of the adenosine triphosphate (ATP) binding site. It blocks the transfer of phosphate groups from ATP to tyrosine residues of the substrates [2]. This causes interruption of the downstream signaling process that leads to cell proliferation, including MAP kinase and Akt. The most common adverse effects include anemia, edema, particularly periorbital, rash/dermatitis, and fatigue. Hepatotoxicity has been rarely reported in anecdotal cases [4-8]. Hepatic injury was manifested as hepatitis, severe hyperbilirubinemia, and focal necrosis [4-8].

Gilbert's syndrome is the most common hereditary cause of mild unconjugated (indirect) hyperbilirubinemia [9]. Individuals with Gilbert's syndrome present with mild, unconjugated hyperbilirubinemia due to impaired glucuronidation by reduced uridine diphosphate glucuronosyltransferase 1A1 (UGT1A1) expression. Various studies have revealed that Gilbert's syndrome has immense clinical and pharmacological importance because the mild hyperbilirubinemia can be mistaken for a sign of occult, chronic, or progressive liver disease as well as this syndrome is associated with lack of detoxification of few drugs, leading to enhanced toxicity, such as irinotecan toxicity [10]. 
Approximately 3-10\% of the population is estimated to have Gilbert's syndrome based on serum bilirubin levels [9]. However, it is also believed as many as $16 \%$ of the total population may have the polymorphism as many people are not tested $[9,10]$. The UGT1A1 polymorphism responsible for the syndrome has been associated with nilotinib, another TKI that targets the BCR-ABL tyrosine kinase, induced hyperbilirubinemia in patients with CML $[11,12]$. FDA has required physicians to check for UGT1A1 polymorphism before starting nilotinib based on the data from earlier studies that showed an increase in hyperbilirubinemia in patients treated with nilotinib who also had the UGT1A1 polymorphism.

Our study extends UGT1A1 molecular analysis to case series in patients who were receiving imatinib for GIST and experienced abnormalities of liver function.

\section{Patients and methods}

The first case is a 44-year-old Caucasian male who presented with recurrent episodes of gastrointestinal bleeding was found to have a mass in the ampulla of Vater. Patient underwent laparoscopic small-bowel resection with primary stapled sideto-side functional end-to-end enteroenterostomy. Pathology was consistent with GIST that was c-KIT positive and DOG-1 positive, and high mitotic rate. In the immediate postoperative period after the small bowel resection, patient was found to have a thrombus in the superior mesenteric vein (SMV) and portal vein (PV) leading to hepatic infarct in segment 7. Patient was treated with lovenox for 4 months. Follow-up MRI after 2 months of lovenox treatment showed no evidence of the thrombi in the SMV and PV as well as the resolution of the perfusion defects seen in the segment 7 of the liver. The patient was offered adjuvant therapy with imatinib $400 \mathrm{mg}$ daily. Liver function tests (LFTs) were monitored twice a month for one month and then every four weeks. Baseline LFTs prior to imatinib initiation were normal. Approximately three months after treatment, a gradual incline in total bilirubin was noticed. Further workup was negative for hepatitis B, C, and HIV. He had minimal alcohol intake (he used to drink one drink once or twice a month but declined any use since his diagnosis) and was not taking any hepatotoxic medication (over-the-counter [OTC], recreational drugs or herbal supplements). Hemolysis parameters were normal as evidenced by normal levels serum lactate dehydrogenase, haptoglobulin, reticulocyte counts were normal as well as lack of abnormal cells on peripheral smear. Repeat MRI showed resolution of the density in the liver and no evidence of biliary obstruction or metastases.

The second case is a 65-year-old Caucasian male who presented with multiple episodes of gastrointestinal bleeding was subsequently found to have a $3-4 \mathrm{~cm}$ submucosal jejunal mass. Pathology was consistent with stage II (pT2pG2N0M0) jejunal GIST (CK positive, and Ki 67 positive) with high mitotic rate. Patient had a laparoscopic jejunectomy followed by a small bowel resection. Adjuvant therapy with imatinib (400 mg daily) was started with baseline normal LFTS prior to initiation. A dose reduction to imatinib $200 \mathrm{mg}$ occurred within two months of treatment due to neutropenia and rash. Patient tolerated the medication without further side effects; however, laboratory results were significant for total bilirubin of $1.9 \mathrm{mg} / \mathrm{dL}$. Hepatitis A, B, C serologies were negative and alcohol intake was limited to 1-2 beers per weekends only. He did not have any evidence of hemolysis as serum lactate dehydrogenase, haptoglobulin, reticulocyte counts were normal and no spherocytosis seen on peripheral blood smear.

\section{Results}

None of these patients had any recent history of foreign travel. Both patients were screened for HAV, HBV, HCV, and HIV. History was ruled out for excessive alcohol intake, use of any hepatotoxic drugs including OTC as well as any herbs. Of utmost importance, the first patient was treated with lovenox for thrombi in the SMV and PV during imatinib therapy, a wise decision made by the oncologist as the FDA has alarmed about drug interactions of imatinib with warfarin, with subsequent resolution of the perfusion defects seen in the segment 7 of the liver.

In addition, assessment by gastroenterology team was carried out who ruled out autoimmune hepatitis (druginduced) based on serology investigations. Both the patient and their caretakers were questioned about any prior history of transient episodes of mild jaundice or skin or eye changes during periods of fasting or febrile illnesses and were denied. All the labs mentioned including $\mathrm{LDH}$, peripheral blood smear, haptoglobin, antibodies (smooth muscle antibodies, anti-mitochondrial antibodies, IgG4), PT, PTT, INR, albumin were performed and were within normal limits. Both patients are lean, no known hyperlipidemia and other risks for factors for nonalcoholic steatohepatitis were identified. Both patients had magnetic resonance cholangiopancreatography and hence imaging ruled out other etiologies such as sclerosing cholangitis, and gallstones. We performed liver biopsy in the first patient while the second patient declined biopsy. No conclusive findings were obtained upon histological assessment. Though LFTs improved initially after dose reduction and omission but normalized, we did rechallenge to further test the association between cause and effect and since hyperbilirubenemia worsened after rechallenge, we ultimately had to stop the medication altogether in the first patient.

The first patient was tested positive for UGT1A1 TA7 $\left({ }^{*} 28\right)$ genotype as homozygous for the TA7 polymorphism. UGT1A1 level confirmed Gilbert's syndrome. Twenty-six weeks from the time of diagnosis of GIST, imatinib was lowered to $200 \mathrm{mg}$ daily for 8 weeks with mild improvement in LFTs. As the total bilirubin continued to rise and peak at $3.0 \mathrm{mg} / \mathrm{dL}$, further dose adjustments were made. He was advised to take only $100 \mathrm{mg}$ daily. LFTs were monitored on regular basis and despite this change his bilirubin continued to elevate. The LFTs continued to rise despite decreasing the dose of imatinib to $100 \mathrm{mg}$ every other day. Therefore, after a detailed decision with the patient regarding the possible hepatotoxicity if he continued the medication versus the risk of recurrence was carried and 


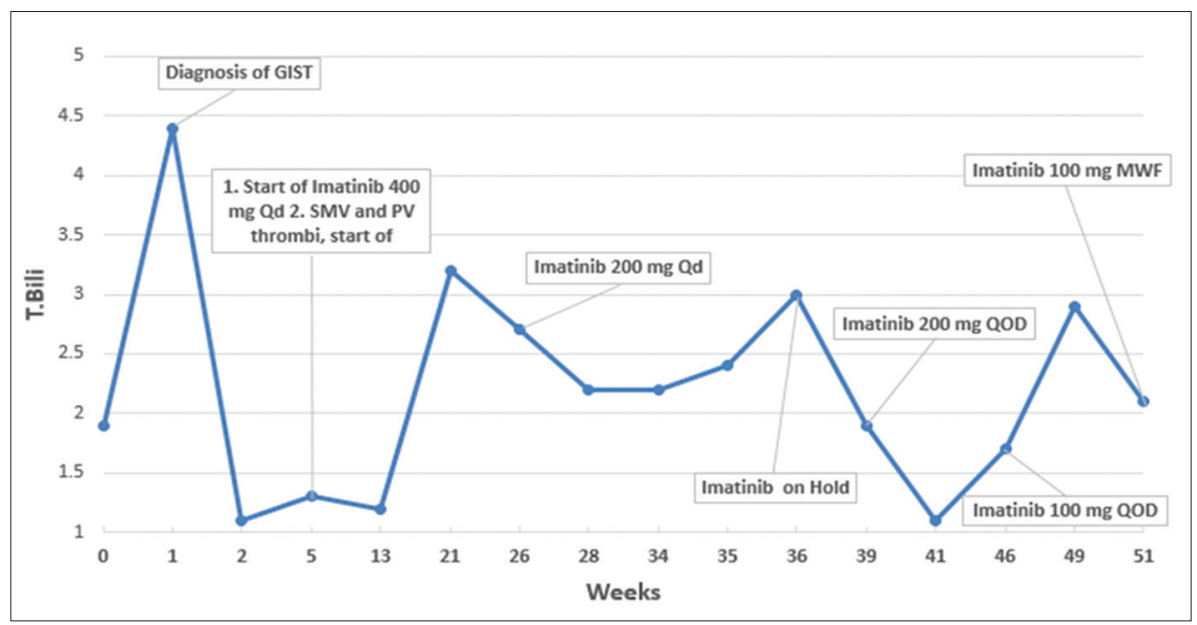

Figure 1 Trend of total bilirubin with the course of imatinib therapy in patient 1

Table 1 Liver function profile of patient \#1 during imatinib therapy

\begin{tabular}{|c|c|c|c|c|c|c|}
\hline Date & AST & LDH & ALK & ALT & T. Bili & Imatinib dose \\
\hline $3 / 2 / 16$ & 32 & 204 & 49 & 51 & 2 & \\
\hline $1 / 6 / 16$ & 27 & 230 & 42 & 28 & 2.1 & \\
\hline $12 / 16 / 15$ & 24 & 188 & 50 & 28 & 2.5 & \\
\hline $9 / 2 / 15$ & 25 & 186 & 45 & 32 & 2.3 & \\
\hline $7 / 1 / 15$ & 25 & 180 & 41 & 28 & 2.8 & Discontinued therapy \\
\hline $6 / 3 / 15$ & 27 & 219 & 42 & 32 & 2.1 & \\
\hline $5 / 18 / 15$ & 28 & 195 & 39 & 33 & 2.9 & Reduce to 100 mg Monday - Wednesday - Friday \\
\hline $4 / 27 / 15$ & 23 & 163 & 40 & 31 & 1.7 & \\
\hline $4 / 1 / 15$ & 25 & 182 & 46 & 29 & 2.9 & Resumed at $100 \mathrm{mg}$ every other day \\
\hline 3/18/15 & 29 & 184 & 49 & 47 & 2.1 & Treatment held \\
\hline $3 / 4 / 15$ & 22 & 170 & 41 & 31 & 1.9 & Resumed at $100 \mathrm{mg}$ every other day \\
\hline 2/11/15 & 24 & 169 & 39 & 30 & 3 & \\
\hline $2 / 4 / 15$ & 26 & 183 & 42 & 30 & 2.4 & \\
\hline $1 / 28 / 15$ & 28 & 213 & 44 & 34 & 2.2 & Treatment held \\
\hline $1 / 14 / 15$ & 25 & 179 & 45 & 33 & 2.2 & \\
\hline $12 / 17 / 14$ & 32 & 169 & 42 & 39 & 2.2 & \\
\hline $12 / 3 / 14$ & 31 & 203 & 45 & 37 & 2.7 & \\
\hline $11 / 21 / 14$ & & & & & & Reduced to $200 \mathrm{mg}$ daily \\
\hline $11 / 5 / 14$ & 32 & 214 & 44 & 39 & 3 & \\
\hline $10 / 29 / 14$ & & & & & 3.2 & \\
\hline $10 / 29 / 14$ & 27 & 230 & 40 & 37 & 3.2 & \\
\hline $10 / 1 / 14$ & 24 & 155 & 40 & 36 & 1.7 & \\
\hline $9 / 3 / 14$ & 29 & 210 & 47 & 47 & 1.2 & \\
\hline $8 / 6 / 14$ & 33 & & 52 & 42 & 1.3 & \\
\hline $7 / 23 / 14$ & 46 & 235 & 61 & 107 & 1.7 & \\
\hline $7 / 9 / 14$ & 39 & 213 & 57 & 61 & 1.3 & Started at $400 \mathrm{mg}$ daily \\
\hline $6 / 18 / 14$ & 18 & 247 & 74 & 33 & 1.1 & \\
\hline $6 / 4 / 14$ & 16 & 137 & 21 & 13 & 4.4 & Thought to be related to DVT as described in the history \\
\hline $6 / 3 / 14$ & 16 & 155 & 21 & 12 & 1.9 & \\
\hline
\end{tabular}

TKI, tyrosine kinase inhibitor; CML, chronic myeloid leukemia; GIST, gastrointestinal stromal tumor; UGT1A1, uridine diphosphate glucoronosyltransferase 1A1; ATP, adenosine triphosphate; LFTs, liver function tests 
imatinib was stopped based on patient's preference. The rise in bilirubin and the correlation of the level over time with the initiation and withdrawal of the drug are highlighted in Fig. 1. Table 1 depicts the LFTs of patient \# 1 during imatinib therapy.

The other patient was tested heterozygous for UGT1A1 TA7 polymorphism. Imatinib dose was decreased to $100 \mathrm{mg}$ daily but due to persistent elevation in the total bilirubin switched to $100 \mathrm{mg}$ every other day. Since then his total bilirubin is under 1.8 but not normal. He is closely monitored and advised to avoid alcohol and any liver affecting medications including over the counter medications. Highlighted in Fig. 2 is the rise in bilirubin for this patient and the correlation of the level over time with the initiation and decreasing doses of the drug. Table 2 depicts the LFTs of patient \#2 during imatinib therapy.

\section{Discussion}

Of utmost notice, previous cases of hepatic dysfunction secondary to imatinib were females and hepatic injury was observed during therapy for CML [4-6] in addition to two cases in GIST patients [7,8]. Moreover, autoimmune hepatitis is also more commonly observed in females [13]. Though autoimmune hepatitis can occur at any age and affects all ethnic groups, Type 1 autoimmune hepatitis is the most common kind seen in North America that mostly affects adolescence or young adulthood, while 70 of them are female. Patients with type 1 autoimmune hepatitis have other concomitant autoimmune disorders, including celiac disease, Crohn's disease, Graves' disease, Hashimoto's disease, proliferative glomerulonephritis, primary sclerosing cholangitis, rheumatoid arthritis, Sjögren's syndrome, lupus erythematosus, type 1 diabetes, or ulcerative colitis. Our patients have no clinical or serological evidence of these autoimmune disorders. Our patients were male and were receiving treatment for GIST.

Individuals with Gilbert's syndrome present with mild, unconjugated hyperbilirubinemia, resulting from impaired
UGT1A1 expression. Approximately $3-10 \%$ of general population is clinically diagnosed to have Gilbert's syndrome based on serum bilirubin levels. Given the prevalence of Gilbert's syndrome and the increasing use of TKI, we encourage physicians to report such cases to increase awareness. Recent data identified that this syndrome has been associated with nilotinib-induced hyperbilirubinemia in patients with CML $[10,11]$. Our study extends the first case series of similar findings in patients receiving imatinib for GIST. Another distinguished factor in our report is the fact that the cases previously reported were consisting of only females and hepatic injury was observed during therapy for CML.

At the annual meeting of the American Society of Hematology, investigators presented their data on same topic but in patients with CML [14]. They investigated a data of 832 patients treated with a TKI for CML at their center and presented the results on 340 patients: imatinib 313, dasatinib 125 , nilotinib 130. They performed genotypic analysis for variations in dinucleotide repeats in the UGT1A1 promoter region. They classified patients into genotypes as 6/6 (homozygous for (TA)6 allele; wild-type), 7/7 (homozygous for (TA)7allele) and 6/7 (heterozygous). The investigators found hyperbilirubinemia in patients with all three genotypes: patients on nilotinib (44\%) versus patients on imatinib (14\%) versus patients on dasatinib (8\%). They concluded that all patients with the $7 / 7$ genotype are susceptible to increases in bilirubin when treated with any of imatinib, dasatinib or nilotinib; however, hyperbilirubinemia is the most commonly seen in patients on nilotinib, suggesting that nilotinib-induced hepatotoxicity may be further added by UGT1A1 inhibition [14].

The previous case reports mentioned both liver toxicity as well as tolerance in patients with liver dysfunction of imatinib while treated mostly for CML but recent reports in GIST patients further underlines the importance of our study [4-8,15-17]. In one of these cases, the findings of liver biopsy and clinical outcome with steroids pointed towards an allergic reaction as a possible cause [5]. Others also suggested the hepatic injury to presumably have been caused by an

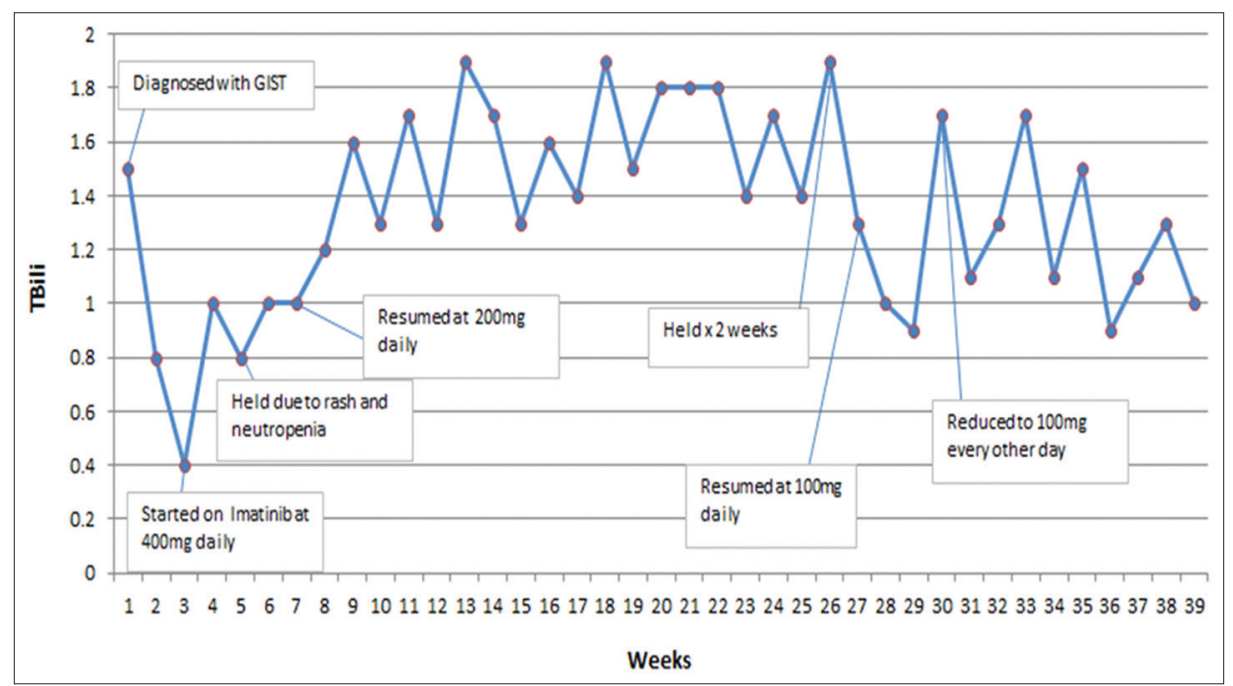

Figure 2 Trend of total bilirubin with the course of imatinib therapy in patient 2 
Table 2 Liver function profile of patient \#2 during imatinib therapy

\begin{tabular}{|c|c|c|c|c|c|c|}
\hline Date & AST & LDH & ALK & ALT & T. Bilirubin & Dose of imatinib \\
\hline $04 / 15 / 16$ & 23 & 162 & 72 & 18 & 1.2 & \\
\hline $02 / 23 / 16$ & 18 & 151 & 73 & 17 & 0.7 & \\
\hline $01 / 12 / 16$ & 29 & 161 & 57 & 22 & 1 & \\
\hline $12 / 1 / 15$ & 23 & 164 & 58 & 19 & 1.3 & \\
\hline $10 / 27 / 15$ & 25 & 314 & 76 & 19 & 1.1 & \\
\hline $09 / 15 / 15$ & 23 & 151 & 50 & 19 & 0.9 & \\
\hline $08 / 5 / 15$ & 24 & 150 & 47 & 18 & 1.5 & \\
\hline 07/08/15 & 28 & 163 & 59 & 20 & 1.1 & \\
\hline 06/09/15 & 30 & 163 & 53 & 24 & 1.7 & \\
\hline 05/06/15 & 25 & 167 & 51 & 20 & 1.3 & \\
\hline 04/01/15 & 21 & 153 & 58 & 16 & 1.1 & \\
\hline $03 / 18 / 15$ & 26 & 170 & 53 & 22 & 1.7 & $100 \mathrm{mg}$ every other day \\
\hline $02 / 18 / 15$ & 26 & 212 & 58 & 24 & 0.9 & \\
\hline $12 / 17 / 14$ & 25 & 159 & 52 & 19 & 1 & $100 \mathrm{mg}$ daily \\
\hline $12 / 03 / 14$ & 29 & 161 & 55 & 22 & 1.3 & \\
\hline $11 / 19 / 14$ & 28 & 175 & 55 & 23 & 1.9 & Held x 2 weeks \\
\hline 09/17/14 & 27 & 178 & 52 & 21 & 1.4 & \\
\hline $07 / 21 / 14$ & 31 & NA & 49 & 25 & 1.7 & \\
\hline 05/07/14 & 31 & 199 & 52 & 20 & 1.4 & \\
\hline $02 / 05 / 14$ & 31 & 183 & 59 & 25 & 1.8 & \\
\hline $10 / 02 / 13$ & 28 & 177 & 56 & 23 & 1.8 & \\
\hline $07 / 29 / 13$ & 36 & 209 & 51 & 26 & 1.8 & \\
\hline $04 / 22 / 13$ & 31 & 147 & 49 & 24 & 1.5 & \\
\hline $03 / 11 / 13$ & 33 & 144 & 48 & 22 & 1.9 & \\
\hline $11 / 26 / 12$ & 31 & 135 & 36 & 24 & 1.4 & \\
\hline $11 / 05 / 12$ & 31 & 143 & 45 & 22 & 1.6 & \\
\hline 10/15/12 & 30 & & 44 & 24 & 1.3 & \\
\hline $06 / 04 / 12$ & 40 & 175 & 43 & 32 & 1.7 & \\
\hline 02/06/12 & 31 & 155 & 53 & 26 & 1.9 & \\
\hline $11 / 28 / 11$ & 31 & 143 & 50 & 24 & 1.3 & \\
\hline 10/24/11 & 30 & 148 & 52 & 22 & 1.7 & \\
\hline $09 / 12 / 11$ & 79 & 191 & 47 & 42 & 1.3 & \\
\hline $07 / 18 / 11$ & 32 & 148 & 53 & 23 & 1.6 & \\
\hline $06 / 13 / 11$ & 29 & 146 & 58 & 23 & 1.2 & \\
\hline $05 / 23 / 11$ & 27 & 150 & 58 & 23 & 1 & \\
\hline 05/09/11 & 25 & 113 & 59 & 22 & 1 & 200 mg daily \\
\hline $04 / 27 / 11$ & 21 & 116 & 77 & 29 & 0.8 & \\
\hline $04 / 22 / 11$ & 19 & 144 & 92 & 26 & 1.1 & Erythematous rash \\
\hline $04 / 13 / 11$ & 39 & 159 & 96 & 38 & 1 & \\
\hline $03 / 16 / 11$ & 17 & 134 & 48 & 14 & 0.4 & \\
\hline 03/07/11 & 22 & & 57 & 24 & 0.8 & 400 mg daily \\
\hline $02 / 26 / 11$ & 23 & 109 & 42 & 21 & 1.5 & \\
\hline
\end{tabular}


Table 3 General guidelines about hepatotoxicity secondary to imatinib Incidence: 6-12\%

Assess liver function before initiation of treatment and monthly
thereafter or as clinically indicated
Gleevec is an inhibitor of CYP3A4 and CYP2D6. Gleevec has
possible interactions with certain other medicines such as warfarin,
erythromycin, and phenytoin, including over-the-counter
medications such as herbal products, as well as grapefruit juice
If Hepatotoxicity observed:
- Withhold if bilirubin $>3 \mathrm{x}$ ULN or ALT/AST $>5 \mathrm{x}$ ULN
- Resume after bilirubin $<1.5 \mathrm{x}$ ULN and ALT/AST $<2.5 \mathrm{x}$ ULN at a
reduced dose (i.e., $400 \mathrm{mg}$ to $300 \mathrm{mg}$;
$600 \mathrm{mg}$ to 400 mg; $800 \mathrm{mg}$ to $600 \mathrm{mg}$ )
- Withhold if severe hepatotoxicity; once resolved, reduce dose
by $25 \%$

autoimmune mechanism [8]. The authors suggested the use of prednisolone when severe hepatic injury suspected to be caused by imatinib, however, such decisions should be only made on the individual patients based on risk and benefit.

No standardized dose guidelines are available. However, the prescribing information (PI) carries the following suggestions:

- Withhold if bilirubin $>3 \mathrm{x}$ upper limit of normal (ULN) or ALT/AST > 5x ULN;

- Resume after bilirubin $<1.5 x$ ULN and ALT/AST $<2.5 x$ ULN at a reduced dose (i.e. $400 \mathrm{mg}$ to $300 \mathrm{mg} ; 600 \mathrm{mg}$ to $400 \mathrm{mg} ; 800 \mathrm{mg}$ to $600 \mathrm{mg}$ ); and

- Withhold if severe hepatotoxicity; once resolved, reduce dose by $25 \%$.

In addition, imatinib is available only in two strengths: $100 \mathrm{mg}$ or $400 \mathrm{mg}$. Since the data is scarce, we decided to be more on conservative side and also kept in mind ease of administration and patient's consent, especially that "severe hepatotoxicity including fatalities may occur" is listed in the PI. Therefore, we used our simplified schedule reducing by $50 \%$ (400 mg - to - $200 \mathrm{mg}$ - to - $100 \mathrm{mg}$ ) tailored to individual patient.

In summary, we report the first two cases of Gilbert's syndrome in patients taking imatinib with the genetic UGT1A1 TA7 or $\left({ }^{\star} 28\right)$ polymorphism. Patients with an unexplained rise in serum bilirubin levels on Imatinib therapy should be screened for the genetic UGT1A1 polymorphisms. It will also be important to know if the side effect profile of TKIs with UGT1A1 is dependent on the genotype of the polymorphism. Recent data has identified that this syndrome is associated with nilotinib, another TKI. Given the prevalence of Gilbert's syndrome and the increasing use of imatinib, we encourage physicians to be aware of this possible toxicity as hepatotoxicity can be fatal if not report such cases to increase awareness (Table 3). If similar cases continue to increase, then the data about imatinib-induced Gilbert's syndrome may prompt a similar FDA requirement for the testing of the UGT1A1 polymorphism as is already done for nilotinib.

\section{References}

1. Druker BJ, Tamura S, Buchdunger E, et al. Effects of a selective inhibitor of the Abl tyrosine kinase on the growth of Bcr-Abl positive cells. Nat Med 1996;2:561-566.

2. Carroll M, Ohno-Jones S, Tamura S, et al. CGP 57148, a tyrosine kinase inhibitor, inhibits the growth of cells expressing BCRABL, TEL-ABL, and TEL-PDGFR fusion proteins. Blood 1997;90:4947-4952.

3. Heinrich MC, Griffith DJ, Druker BJ, et al. Inhibition of c-kit receptor tyrosine kinase activity by STI 571, a selective tyrosine kinase inhibitor. Blood 2000;96:925-932.

4. AlSobhi E, Zahrani Z, Zevallos E, Zuraiki A. Imatinib-induced immune hepatitis: case report and literature review. Hematology 2007;12:49-53.

5. Ikuta $K$, Torimoto $Y$, Jimbo J, Inamura J, Shindo $M$, Sato $K$, Tokusashi Y, Miyokawa N, Kohgo Y. Severe hepatic injury caused by imatinib mesylate administered for the treatment of chronic myeloid leukemia and the efficacy of prednisolone for its management. Int J Hematol 2005;82:343-346.

6. Ohyashiki K, Kuriyama Y, Nakajima A, Tauchi T, Ito Y, Miyazawa H, Kimura Y, Serizawa H, Ebihara Y. Imatinib mesylate-induced hepatotoxicity in chronic myeloid leukemia demonstrated focal necrosis resembling acute viral hepatitis. Leukemia 2002;16:2160-2161.

7. Yachoui R. Early onset imatinib mesylate-induced hepatotoxicity in a patient with gastrointestinal stromal tumors. Am J Ther 2014;21:e148-e150.

8. Aliberti S, Grignani G, Allione P, et al. An acute hepatitis resembling autoimmune hepatitis occurring during imatinib therapy in a gastrointestinal stromal tumor patient. Am J Clin Oncol 2009;32:640-641.

9. Rasool A, Sabir S, Ashlaq M, Farooq U, Khan MZ, Khan FY. Gilbert's syndrome - a concealed adversity for physicians and surgeons. J Ayub Med Coll Abbottabad 2015;27:707-710.

10. Etienne-Grimaldi MC, Boyer JC, Thomas F, et al; Collective work by Groupe de Pharmacologie Clinique Oncologique (GPCOUnicancer); French Réseau National de Pharmacogénétique Hospitalière (RNPGx). UGT1A1 genotype and irinotecan therapy: general review and implementation in routine practice. Fundam Clin Pharmacol 2015;29:219-237.

11. Singer JB, Shou Y, Giles F, et al. UGT1A1 promoter polymorphism increases risk of nilotinib-induced hyperbilirubinemia. Leukemia 2007;21:2311-2315.

12. Chen SP, Poon WT, Mak CM, et al. Application of pharmacogenetics: UGT1A1 ${ }^{\star} 28$ and nilotinib-induced unconjugated hyperbilirubinaemia in a patient with chronic myeloid leukaemia. Pathology 2011;43:273-274.

13. Manns MP, Czaja AJ, Gorham JD, et al. Diagnosis and management of autoimmune hepatitis. Hepatology 2010;51:1-31.

14. Bahk J, Claudiani S, Szydlo RM, et al. The Association of Gilbert's Syndrome with Hyperbilirubinaemia Occurring on Any of Imatinib, Dasatinib and Nilotinib in Patients with Chronic Myeloid Leukaemia (CML). ASH Annual meeting Poster II. Session: 632.

15. Ramamoorthy SK, Pandita R, Prakash A, Ramaswamy NV, Al Bahar S. Safety of imatinib in chronic myeloid leukemia in blastic crisis presenting as cholestatic jaundice. Acta Haematol 2007;118:141-145.

16. Ridruejo E, Cacchione R, Villamil AG, Marciano S, Gadano AC, Mandó OG. Imatinib-Induced Fatal Acute Liver Failure. World J Gastroenterol 2007;13:6608-6111.

17. Tonyali O, Coskun U, Yildiz R, et al. Imatinib mesylate-induced acute liver failure in a patient with gastrointestinal stromal tumors. Med Oncol 2010;27:768-773. 